\title{
Paravesicular Paraganglioma manifesting as Chronic Hypertension and Hypertensive Crisis
}

\author{
${ }^{1}$ Yi Cai, ${ }^{2}$ Anna Kundel, ${ }^{3}$ William Young, ${ }^{4}$ Geoffrey Thompson
}

\begin{abstract}
We describe a woman with a rare extra-adrenal pheochromocytoma who presented with a chronic hypertension and a hypertensive crisis initiated by an invasive cardiac catheterization procedure. We present a case report, review the relevant literature, and discuss management strategies. A 49-year-old woman with a 4-year history of hypertension experienced a hypertensive crisis following a catheterization procedure. Evaluation of the episode led to identification of a catecholaminesecreting pelvic paraganglioma as confirmed by 24-hour urine fractioned metanephrines and catecholamines as well as magnetic resonance imaging. The patient was carefully pharmacologically prepared for surgical resection. Paragangliomas may exhibit $30 \%$ inheritability, and the patient was counseled on genetic testing for early diagnosis of tumor in family members. Catecholamine-secreting paragangliomas are rare tumors that may present as hypertensive emergencies. Diagnosis relies on biochemical testing followed by imaging to avoid crisis induced by invasive procedures. Surgical excision is the treatment of choice, and pharmacological preparation for surgery begins at least 1 week prior to the procedure to ensure adequate adrenergic blockade. Following treatment for a paraganglioma, genetic testing should be recommended for early identification of affected family members.
\end{abstract}

Keywords: Paraganglioma, Hypertensive crisis, Extra-adrenal pheochromocytoma.

How to cite this article: Cai Y, Kundel A, Young W, Thompson G. Paravesicular Paraganglioma manifesting as Chronic Hypertension and Hypertensive Crisis. World J Endoc Surg 2014; 6(2):85-88.

Source of support: Nil

Conflict of interest: None

\section{CASE REPORT}

A 49-year-old woman with a 4-year history of hypertension presented with episodic chest pain associated with diaphoresis, nausea and emesis. The chest pain prompted a cardiac evaluation that included coronary angiography

\footnotetext{
${ }^{1}$ Medical Student, ${ }^{2}$ Fellow, ${ }^{3,4}$ Professor

${ }^{1}$ Department of Gastroenterologic and General Surgery, Mayo Clinic College of Medicine, Rochester, Minnesota, United States

${ }^{2,4}$ Department of Surgery, Mayo Clinic, Rochester, Minnesota United States

${ }^{3}$ Department of Endocrinology and Metabolism, Mayo Clinic Rochester, Minnesota, United States

Corresponding Author: Geoffrey Thompson, Professor Department of Surgery, Mayo Clinic, Rochester, Minnesota United States, Phone: 5072844499, e-mail: thompson. geoffrey@mayo.edu
}

during which the patient had uncontrolled hypertension requiring an admission to the intensive care unit. Evaluation at that time revealed elevated 24-hour urine normetanephrine at $15,000 \mu \mathrm{g}$ (normal, $<451 \mu \mathrm{g}$ ), indicating the presence of a catecholamine-secreting tumor. Magnetic resonance imaging (MRI) scan with gadolinium enhancement identified a $7.5 \mathrm{~cm}$ paravesicular mass located posterior to the right ovary, lateral to the uterus, and anterior to the right piriformis muscle (Figs 1A and B). The patient denied pelvic pain or urinary complaints. She was referred to Mayo Clinic for further management.

At our institution, the plasma normetanephrine concentration was $54 \mathrm{nmol} / 1$ (normal, $<0.9 \mathrm{nmol} / \mathrm{l}$ ) and the patient was begun on $\alpha$-adrenergic blockade (phenoxybenazamine).
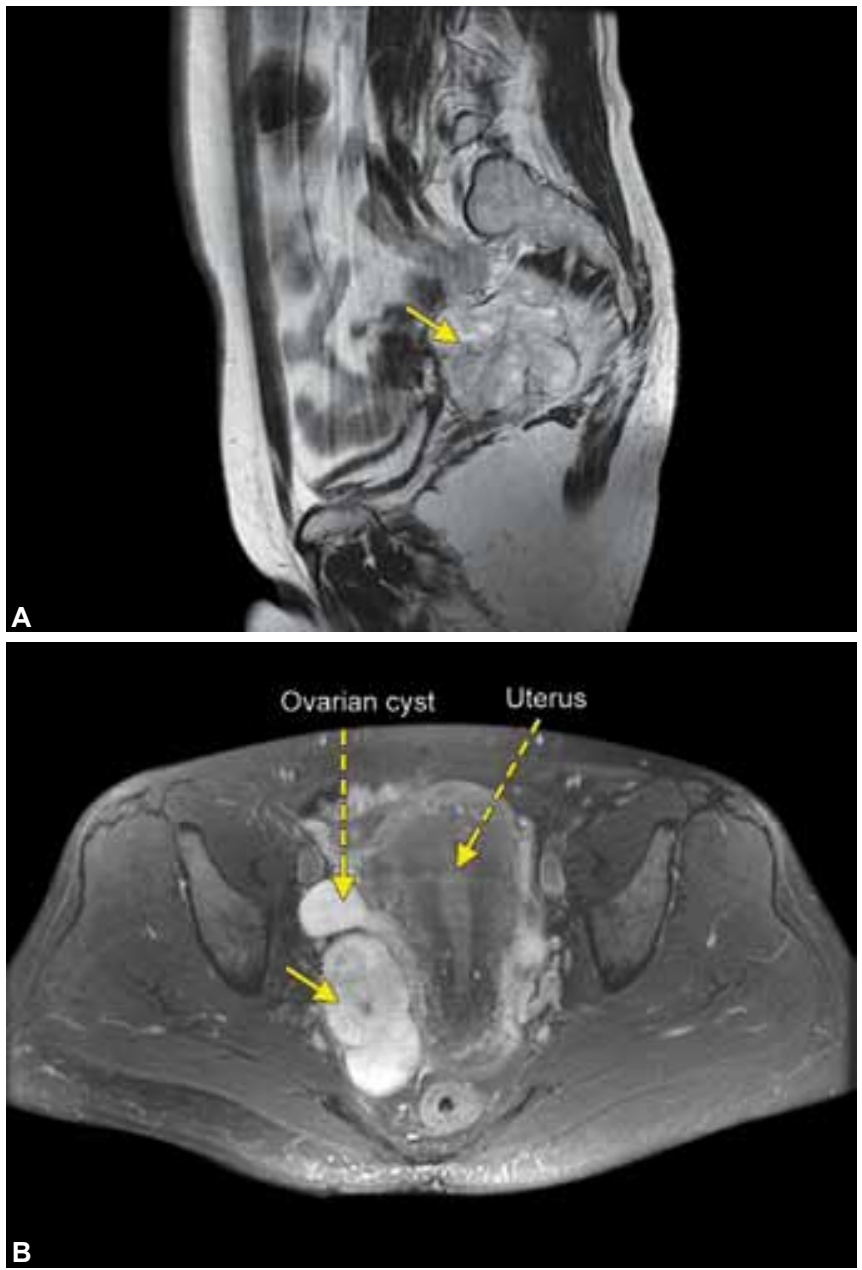

Figs 1A and B: T2 weighted MRI (sagittal image on upper and axial image on lower) revealed a $7.5 \mathrm{~cm}$ paravesicular mass (solid arrow). The mass is posterior to the right ovary, lateral to the uterus, and anterior to the right piriformis muscle 


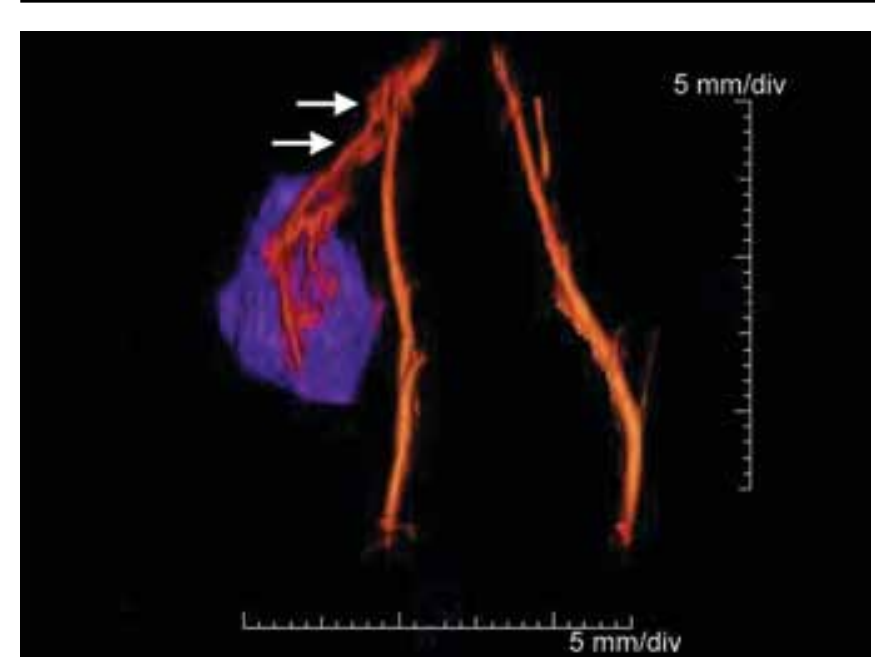

Fig. 2: MR angiogram. 3D volume rendered image from an MR angiogram, revealing arterial supply to the paraganglioma from the right internal iliac artery (arrows indicate new vasculature). Arrows are pointing to the internal iliac artery

The addition of $\beta$-adrenergic and calcium-channel blockers were required for adequate heart rate and blood pressure control. MR angiography was employed to better delineate vascular involvement and revealed that the tumor was perfused by branches of the right internal iliac artery (Fig. 2).
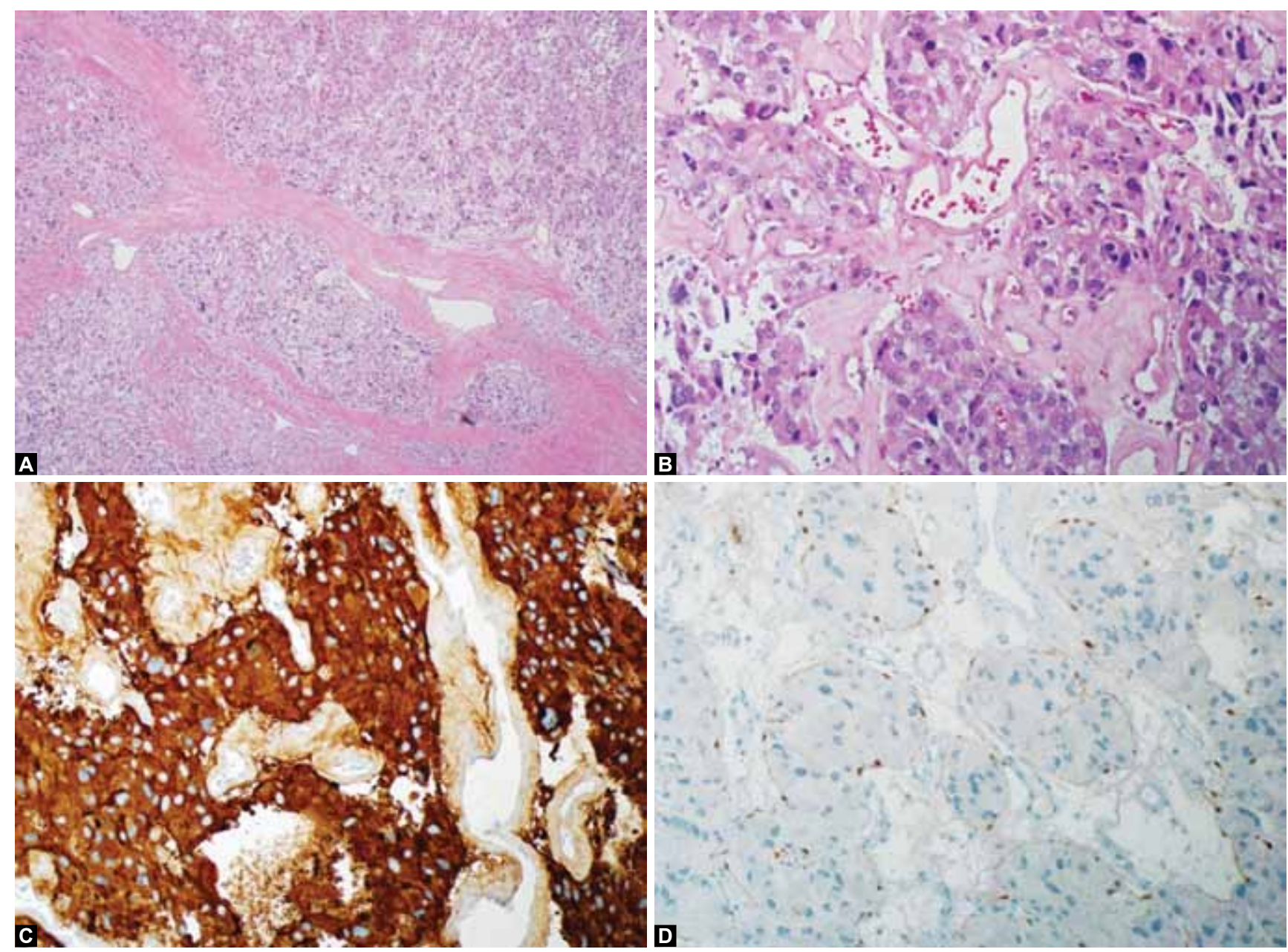

Figs 3A to D: Pathology. Hematoxylin and Eosin ( $\mathrm{H}$ and $\mathrm{E})$ reveals bands of fibrosis entrapping lobules of tumor cells $(\mathrm{A} ; 100 \mathrm{x})$ and the alveolar (Zellballen) arrangement of chief cells with tumor cell nests in a highly vascular stroma (B; 400x). Immunohistochemical stain confirms the neuroendocrine nature of this neoplasm (C: Synaptophysin, 200x; D: S-100, 200x)
${ }^{123}$ I-metaiodobenzylguanidine scintigraphy (MIBG) scan indicated a localized mass without metastasis. Genetic testing was offered and extensively discussed with the patient and family members but was declined.

At the time of surgery, arterial and central lines were placed for optimal blood pressure monitoring and control. To guide in the identification of the right ureter during exploration, a right ureteral stent was placed cystoscopically. The paraganglioma was approached through a midline abdominal incision and dissected away from the adherent vaginal, bladder and rectal walls. The right internal iliac vessels were sacrificed, taking care to preserve the closely

The tumor was a $9.7 \mathrm{~cm}$ well-encapsulated soft-tissue mass. Diagnosis of paraganglioma was confirmed by histology, showing typical nesting Zellballen appearance on hematoxylin and eosin staining and by presence of synaptophysin and S-100 positivity on immunohistochemistry (Figs $3 \mathrm{~A}$ to $\mathrm{D})$. No lymphatic invasion was noted.

Her postoperative course was complicated by a pneumothorax resulting from preoperative line placement. This involved right ureter. 
was successfully managed with closed tube thoracostomy. She recovered well after surgery and was released from the hospital on postoperative day four. Upon discharge, her plasma fractionated metanephrines were within the reference ranges, and she was normotensive off all antihypertensive medications.

\section{DISCUSSION}

Paraganglia are derived from neural crest cells and are part of both the sympathetic and parasympathetic nervous systems that secrete catecholamines and other neurotransmitters in response to stress. Most catecholamine-secreting tumors are intra-adrenal (pheochromocytomas), and only 15\% are paragangliomas. While most paragangliomas of the parasympathetic system are nonfunctional and are located in the skull base and neck, sympathetic paragangliomas are more likely to be functional (up to $85 \%$ ) and occur most commonly in the abdominal para-aortic region. ${ }^{1}$ Of all paragangliomas, $75 \%$ are located in the superior and inferior abdominal paraaortic areas, $10 \%$ in the urinary bladder, $10 \%$ in the thorax, and $5 \%$ in the skull base and neck. ${ }^{2}$

Symptoms of functional paragangliomas are due to catecholamine excess, presenting as tachycardia, diaphoresis, and hypertension that may result in paroxysmal headaches or hypertensive crises. ${ }^{3}$ With regard to pelvic paragangliomas, most described cases have originated within the urinary bladder wall and may present with hypertension, headache, hematuria and micturition syncope. ${ }^{4}$ Rare cases have been described in association with the ureter, ${ }^{5}$ vagina, ${ }^{6}$ ovaries ${ }^{7}$ and prostate. ${ }^{8}$ Many of these patients were identified incidentally on imaging, or, if symptoms were present, they varied from resistant hypertension and occasional palpitations to syncope on micturition or mass effect. The patient presented herein illustrates a rare case of a sympathetic, catecholamine-secreting pelvic paraganglioma adjacent to the vaginal, bladder, and rectal walls and was suspected during a cardiac catheterization-triggered hypertensive crisis.

When a catecholamine-secreting tumor is suspected, the evaluation should begin with biochemical testing, which includes 24-hour urine fractioned metanephrines and catecholamines. Plasma fractionated metanephrines are less specific, but are useful for diagnosis in highly suspect patients and for postoperative monitoring. ${ }^{9}$ If clinical suspicion is low, one must remember the most common causes for increased levels of fractionated catecholamines and metanephrines, which include medications (e.g. tricyclic antidepressants, antipsychotics, levodopa and sympathomimetics) and physical stress (e.g. hypertensive stroke, myocardial infarction). Once the biochemical diagnosis is established, computed tomography (CT) or MRI scan of the abdomen and pelvis should be employed for localization. MRI is not preferred over CT, but may help determine vascular anatomy adjacent to the paraganglioma. MR angiography can further aid in assessing vascular involvement, and MIBG and ${ }^{18} \mathrm{~F}$-fluorodeoxyglucose positron emission tomography can help identify sites of metastatic disease.

Surgical excision is the treatment of choice for these tumors. Good control of blood pressure is required prior to excision and is achieved via adrenergic blockade. Phenoxybenzamine (a nonselective $\alpha$-adrenergic blocker) should be started at least 7 days preoperatively along with increased salt and fluid intake for volume expansion. This preoperative preparation lessens the risk of intraoperative hypertension and helps to avoid postoperative hypotension after tumor resection. ${ }^{10}$ Once $\alpha$-adrenergic blockade is achieved, $\beta$-adrenergic as well as calcium-channel blockers may also be employed for heart rate and further blood pressure control. The tyrosine hydroxylase inhibitor, alpha-methylparatyrosine (metyrosine), can be used in selected cases when blood pressure cannot be controlled with traditional agents or if a complicated resection is anticipated.

Follow-up consists of clinical evaluation and yearly plasma or urine fractionated metanephrines levels. Germline mutation testing should be discussed and offered to all patients with paragangliomas. Approximately 30\% of paragangliomas have a genetic component, related most commonly to familial paraganglioma syndromes due to a mutation in the succinate dehydrogenase (SDH) tumor suppressor gene subunits SDHB, SDHD, SDHC, SDHAF2 and SDHA. SDHB mutations are most commonly associated with pelvic paragangliomas and exhibit greater than $50 \%$ risk of malignancy. ${ }^{11}$ If germline mutations are uncovered in a patient, early screening of kindred members may reduce the risk of morbidity and mortality.

Catecholamine-secreting paragangliomas are rare tumors that may present as hypertensive emergencies during an unrelated procedure. If a tumor is found incidentally within the pelvis, paraganglioma should be in the differential diagnosis and can easily be ruled out with biochemical testing, thus avoiding a crisis. Surgical removal is the treatment of choice, but careful preoperative pharmacologic management is imperative for successful excision. Due to close association with the often malignant SDHB mutation, genetic testing is recommended for the early identification of this tumor in family members.

\section{REFERENCES}

1. Erickson D, Kudva YC, Ebersold MJ, et al. Benign paragangliomas: clinical presentation and treatment outcomes in 236 patients. J Clin Endocrinol Metab 2001;86(11):5210-5216.

2. McNicol AM. Update on tumours of the adrenal cortex, phaeochromocytoma and extra-adrenal paraganglioma. Histopathology 2010;58(2):155-168. 
3. Young WF Jr. Paragangliomas: clinical overview. Ann NY Acad Sci 2006;1073:21-29.

4. Beilan J, Lawton A, Hajdenberg J, et al. Pheochromocytoma of the urinary bladder: a systematic review of the contemporary literature. BMC Urol 2013;13(1):22.

5. Awasthi NP, Kumari N, Krishnani N, et al. Functional paraganglioma of ureter: an unusual case. Indian J Pathol Microbiol 2011;54(2):405-406.

6. Akl MN, Naidu SG, McCullough AE, et al. Vaginal paraganglioma presenting as a pelvic mass. Surgery 2010;147(1):169-171.

7. McCluggage WG, Young RH. Paraganglioma of the ovary: report of three cases of a rare ovarian neoplasm, including two exhibiting inhibin positivity. Am J Surg Pathol 2006;30(5):600-605.
8. Padevit C, John H, Gunz A, et al. Micturition syncope due to paraprostatic pheochromocytoma. Urol Int 2005;74(3):276-277.

9. Sawka AM, Jaeschke R, Singh RJ, et al. A comparison of biochemical tests for pheochromocytoma: measurement of fractionated plasma metanephrines compared with the combination of 24-hour urinary metanephrines and catecholamines. J Clin Endocrinol Metab 2003;88(2):553-558.

10. Kinney MA, Warner ME, vanHeerden JA, et al. Perianesthetic risks and outcomes of pheochromocytoma and paraganglioma resection. Anesth Analg 2000;91(5):1118-1123.

11. Fishbein L, Nathanson KL. Pheochromocytoma and paraganglioma: understanding the complexities of the genetic background. Cancer Genetics 2012;205(1-2):1-11. 